\title{
Life on the fast lane: the burst mode at the VLT at present and in the future
}

\author{
Andrea Richichi ${ }^{1}$, Octavi Fors ${ }^{2}$, Elena Mason $^{3}$, Marco Delbó ${ }^{4}$, Jörg \\ Stegmaier $^{1}$, and Gert Finger ${ }^{1}$ \\ 1 European Southern Observatory, Garching, Germany arichich@eso.org \\ 2 Departament d'Astronomia i Meteorologia, Barcelona, Spain of ors@am.ub.es \\ 3 European Southern Observatory, Paranal, Chile emason@eso.org \\ 4 Observatoire de la Côte d'Azur, Nice, France delbo@oca.eu \\ 5 European Southern Observatory, Garching, Germany jstegmai@eso.org \\ ${ }^{6}$ European Southern Observatory, Garching, Germany gfinger@eso.org
}

Summary. The recent implementation of the high-speed burst mode at the ISAAC instrument on UT1, and its propagation to other ESO instruments, has opened the door to observational capabilities which hold the potential for a wealth of novel results. In the ELT era, when the accent will likely be on lengthy programs aimed at the best sensitivity and angular resolution, the VLT telescopes could continue to play a significant and largely unique role by performing routinely observations of transient events at high temporal resolution. In our contribution, we provide details on two such kinds of observations, namely lunar occultations of stars and of asteroids. For the first ones, we report on two passages of the Moon in regions with high stellar density as the Galactic Center. The VLT-UT1 telescope was used for the first time to record successfully 53 and 71 occultations on March 22 and August 6, 2006, with an angular resolution of 0.5-1 milliarcsecond and $K \sim 12.5$ limiting magnitude. We note that the angular resolution is superior to that achieved at present by Adaptive Optics on any telescope, and also superior to that foreseen for the ELT at the same wavelength. LO are also very efficient in terms of telescope time. We present some of the results, including the discovery of close binaries, and the detection and study of compact circumstellar components of cool giants, AGB stars and embedded IR sources.

Concerning asteroidal occultations, we aim at observations starting in P80 which would permit high-accuracy, direct determinations of asteroid sizes for bodies larger than $\approx 50 \mathrm{~km}$. This is a critical information to improve our understanding of the physical properties of these bodies. It will allow us an independent, crucial calibration of the indirect techniques commonly used to derive estimates of asteroid sizes and albedo, namely radiometry (Harris \& Lagerros [4]) and polarimetry (Cellino et al [1], and references therein). Lunar occultations can be used also to detect asteroid binary systems, which have been found recently to be not very rare. Binary systems are invaluable to estimate asteroid masses and densities, parameters that are at present very poorly known. 


\section{Scientific motivations}

Our main scientific driver are lunar occultations of stellar sources, and we provide here some details on the goal and the technical requirements. Later we will mention other astrophysical topics which also require fast, or very fast, photometry. Lunar occultations (LO) represent a very powerful and yet extremely simple method to obtain high angular resolution information on sources covered by the Moon during its apparent motion as seen from a specific site. The obvious disadvantages of $\mathrm{LO}$ are that we cannot choose the targets at will, and that they are fixed time events. Fig. 1 illustrates the basic geometry of an occultation event. The position angle (PA) defines the scan direction of the lunar limb across the source. The local limb slope $\psi$ can influence this by a few degrees. The contact angle CA defines the effective speed $V_{P}$ of the limb motion, which can be significantly slower than the apparent lunar motion $\mathrm{V}_{\mathrm{M}}$, especially for $\mathrm{LO}$ approaching grazing conditions. The parameter $\mathrm{V}_{\mathrm{P}}$, in conjunction with the observing wavelength, defines the rate of the diffraction fringes. The model light curve of Fig. 1 has been generated for a typical speed of $0.5 \mathrm{~m} / \mathrm{s}$, in a broad-band $\mathrm{K}$ filter.
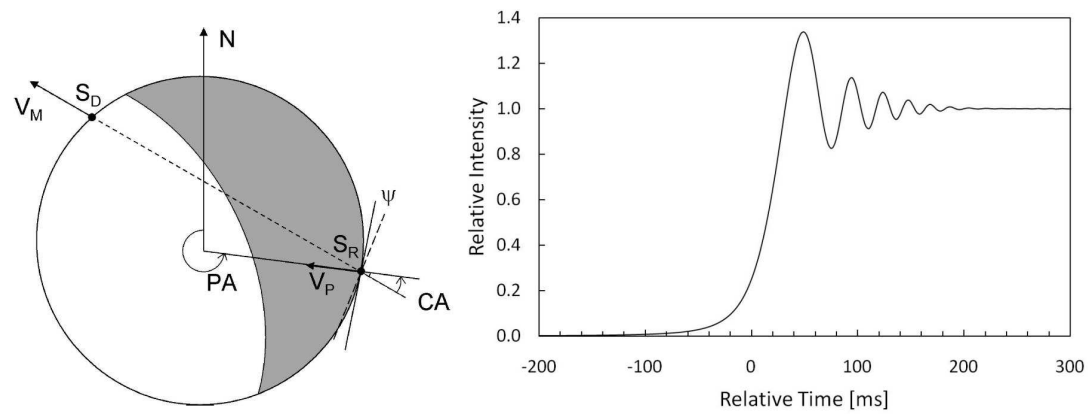

Fig. 1. Left: Scheme of a lunar occultation event. Symbols are explained in the text. right: A typical (near-IR) model occultation light curve. Note the temporal scale of the diffraction fringes, which requires high speed photometry for proper sampling.

The detailed analysis of the diffraction light curve of a LO is outside the scope of this contribution, but we mention that it is rather simple and relatively quick. It can recover information on the occulted source with angular resolutions of $\approx 1$ mas, and can even be used to reconstruct brightness profiles of complex sources without any model-dependent parameters. These characteristics make LO competitive with other high-angular resolution methods such as AO or long-baseline interferometry. However, the light curve must be sampled at millisecond rates. This was easily achieved with simple photometers: single pixel InSb detectors have been routinely used for this work in the near-IR. On the other hand, these photometers presented the disadvantage of integrating the light over the whole diaphragm, thus resulting in 
added noise from the rather high background normally encountered during LO observations.

The progressive introduction of array detectors has significantly changed the landscape of near-IR instrumentation. Nowadays, almost all telescopes of medium to large size are equipped with such detectors, which emphasize area and read-out noise (RON) but, with the possible exception of specialized detectors for wavefront sensing, are usually slow to read-out. However, suitable compromises can be realized by reading out fast only parts of the arrays. A comparison of the two approaches was made by Richichi [7], and we refer to this paper for an analytic and quantitative comparison of the signalto-noise ratio (SNR) for the photometer and the array cases under various combinations of telescope size, source brightness and background intensity. The comparison included $4 \mathrm{~m}$ and $8 \mathrm{~m}$ telescopes: while these latter might have seemed a remote possibility ten years ago, LO have now been observed on one of the $8.2 \mathrm{~m}$ VLT telescopes equipped with the ISAAC instrument in the so-called burst mode (Richichi et al. [8]).

Presently, the burst mode is limited to sampling times of 3-5 ms. This is suitable for LO work, as well as for other applications of moderately fast photometry such as occultation and transit phenomena in the solar system, transient and oscillatory phenomena in stars and binary stars, and some aspects of the highly time variable properties of white dwarfs, neutron stars and pulsars - where of course sensitivity is the main barrier. A list of high-time resolution astrophysical topics and available instruments has recently been presented by Redfern \& Ryan [5].

\subsection{The burst mode of ISAAC}

A fast readout mode on a user-defined subarray has recently been implemented, tested and commissioned on the Aladdin detector of the ISAAC instrument [8]. This burst mode is optimized for speed, at the expense of data organization which needs to be performed offline. The raw data consist in a sequential write of successive reads of the subarray, i.e. they are double the size of the standard FITS cubes and do not have a FITS structure. A similar mode, so-called Fastjitter, can achieve standard data organization but is several times slower. The main characteristics of the burst mode are listed in Table 1.

We have used the burst mode on the occasion of two series of lunar occultations in crowded regions near the galactic center, on March 22 and August 6,2006 . In particular for the second event, we were able to observe 71 occultations over a period of few hours. For experienced observers, it is possible to offset the telescope, start the observation, record the data in a total of about two minutes. Preliminary results from these runs have been reported by Fors et al. [2]. Among them, we mention 7 small separation binaries, 5 angular diameters, and 4 sources with extended circumstellar emission. An example is shown in Fig. 2. 
Table 1. Main parameters of the ISAAC burst mode

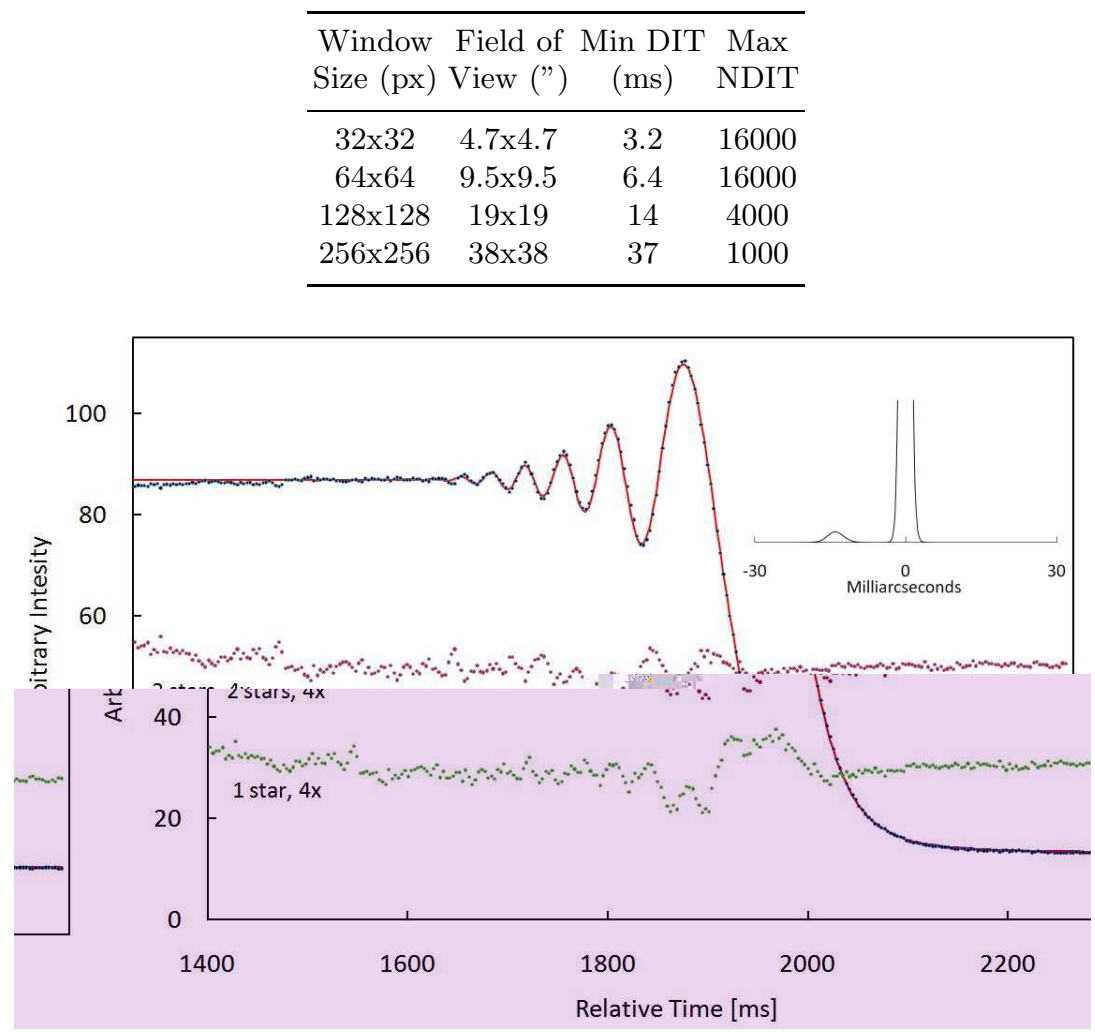

Fig. 2. An anonymous star observed on August 6, 2006 (2MASS 17524903-2822586), shown to be a small separation, low contrast binary. The main panel shows the data, the best fit with a binary star, and two sets of fit residuals: one for a single source and one for the binary model. The residuals are offset by arbitrary amounts and enlarged for clarity. The inset is a profile reconstruction using a model independent method (Richichi [6]). The companion has a projected separation of $12.8 \pm 2$ mas, and a brightness ratio of 1:35 (magnitudes of the two components are $\mathrm{K}=5.23$ and $\mathrm{K}=9.12$, respectively).

The study by Richichi [7] predicted that an $8 \mathrm{~m}$ telescope equipped with a near-IR array detector would reach between $\mathrm{K}=12$ and 14 mag, depending on the lunar phase and background, with an integration time of $12 \mathrm{~ms}$ at $\mathrm{SNR}=10$. The preliminary results with ISAAC reported by Richichi et al. [8] show a limiting magnitude $\mathrm{K} \approx 12.5$ at $\mathrm{SNR}=1$ and $3 \mathrm{~ms}$ integration time. Within the uncertainty of the lunar background, the results are in perfect agreement with the decade-old prediction. Thanks also to the introduction of massive predictions based on IR survey catalogues such as 2MASS and of automated data pipelines based on new methods of light curve characteriza- 
tion based on the wavelet theory (Fors et al. [3]) it is now possible to imagine programs of routine LO observations also at the VLT. We have prepared one such program as a filler for brief unused telescope times, which has been approved for P80 and has been resubmitted for P81.

\subsection{Asteroid occultations}

The interest in $\mathrm{LO}$ of asteroids stems from the possibility to derive the sizes of these bodies from the duration of the occultation event. We also note that for objects with angular sizes of $\approx 20$ mas or larger, the LO light curves can be analyzed by simple geometrical (rather than diffraction) optics. In these cases, details of the brightness profile, connected with the distribution of albedo and irregularities on the surface, can be measured. Moreover, the technique is ideally suited to discover new binary systems. Asteroids are moving objects and the prediction of the epoch and geometry of their lunar occultations, along with the visibility of the events from Paranal, must be calculated for each of object. The $\mathrm{K} \approx 12.5$ limiting magnitude of the high-speed burst mode of ISAAC on the UT1, roughly scales to a limiting asteroid magnitude of $\mathrm{V} \approx 14.5$. Using a computer code to predict the lunar occultations involving the first 5,000 numbered asteroids (asteroids with number $>5,000$ are in general likely to have $\mathrm{V}>14.5$ ), we find that there are about 40 lunar occultations of asteroids observable from Paranal every year. The unique combination of ISAAC and UT1 makes it possible to observe occultations events of asteroids with diameters in the range between 100 and $50 \mathrm{~km}$ in the Main Belt and obtain direct determination of their sizes. In this size range, asteroids display angular extensions between 50 and 100 mas. At the average lunar proper motion their disappearance last $100 \mathrm{~ms}$ (minimum). By tuning the integration time to yield SNR $>10$ the sizes of these bodies can be determined with the unprecedented accuracy of some percent.

\section{References}

1. A. Cellino, R.G. Hutton, M. di Martino, P. Bendjoya, I. Belskaya, E.F. Tedesco: Icarus 179, 304 (2005)

2. O. Fors, A. Richichi, E. Mason, J. Stegmaier, T. Chandrasekhar: Highlights of Spanish Astrophysics IV, Figueras et al. Eds. (2007)

3. O. Fors, A. Richichi, X. Otazu, J. Nuñez: $A \& A$ submitted, arXiv:0711.0537 (2007)

4. A.W. Harris, J.S.V. Lagerros: Asteroids III, W.F. Bottke, A. Cellino, P. Paolicchi, R.P. Binzel (Eds.), Univ. of Arizona Press, Tucson, AZ, 205 (2002)

5. M. Redfern, O. Ryan: Towards the European ELT, Marseille (2006)

6. A. Richichi: $A \& A, \mathbf{2 2 6}, 366$ (1989)

7. A. Richichi: IAU Symp. 158 Very High Angular Resolution Imaging, p 71 (1997)

8. A. Richichi, O. Fors, E. Mason, J. Stegmeier: The Messenger, 126, 24 (2006) 